\section{Factores pronósticos en colitis ulcerosa de reciente diagnóstico}

\author{
GONZALO PIZARRO ${ }^{1,2, a}$, RODRIGO QUERA ${ }^{3}$, \\ CAROLINA FIGUEROA ${ }^{3}$
}

\section{Prognostic factors of ulcerative colitis at the moment of diagnosis}

The clinical presentation of ulcerative colitis at the moment of diagnosis is variable, and its clinical course is difficult to predict. It can range from a quiescent to a refractory chronic course that may require hospitalization and surgical procedures. It can also have complications such as colorectal cancer. In this review we discuss the role of demographic, clinical, endoscopic, histological and associated factors, which can help to predict the clinical course of the disease at the moment of diagnosis, and to individualize therapy according to this clinical risk. Accurate identification of patients with a newly diagnosed ulcerative colitis who are at high risk of an unfavorable outcome is still a challenge. However, an effective evaluation allows an early diagnosis, a timely and effective treatment.

(Rev Med Chile 2017; 145: 1319-1329)

Key words: Colitis, Ulcerative; Gastrointestinal Diseases; Inflammatory Bowel Diseases; Proctocolectomy, Restorative.
'Servicio de Gastroenterología, Hospital Barros Lucos Trudeau. Santiago, Chile. ${ }^{2}$ Servicio de Gastroenterología, Clínica Tabancura. Santiago,

Chile.

${ }^{3}$ Servicio de Gastroenterología, Programa Enfermedad Inflamatoria Intestinal Clínica Las Condes. Santiago, Chile.

aBecado de Enfermedad Inflamatoria Intestinal, Universidad de Chile - Clínica Las Condes.

Los autores no declaran conflicto de interés.

El trabajo no recibió

financiamiento externo.

Recibido el 7 de marzo de 2016, aceptado el 23 de noviembre de 2016.

Correspondencia a: Dr. Gonzalo Pizarro Jofré Gran Avenida José Miguel Carrera 3122, San Miguel, Región Metropolitana.

gonzalo.pizarro.jofre@gmail.com
$\mathrm{L}$ a colitis ulcerosa (CU) es una patología inflamatoria crónica, limitada al colon y recto. Aunque su etiología es desconocida, sujetos genéticamente predispuestos parecen tener una respuesta inmune descontrolada contra el medio ambiente y la microbiota intestinal, lo que determina inflamación y daño en la mucosa ${ }^{1}$. Su incidencia ha aumentado en países industrializados, lo que sugiere que factores ambientales jugarían un rol en su etiopatogenia ${ }^{2}$. En Chile, un estudio señala que la tendencia de la CU y la enfermedad de Crohn han aumentado progresivamente durante la última década ${ }^{3}$.

La presentación clínica al diagnóstico es variable, siendo difícil predecir su evolución, pudiendo ir desde una enfermedad quiescente a un curso crónico refractario, que determina, eventualmente, necesidad de hospitalizaciones, cirugía o complicaciones como el cáncer colorrectal (CCR), comprometiendo finalmente la calidad de vida ${ }^{4}$.

Se reconocen cuatro formas evolutivas: 1) ac- tividad inicial grave con posterior remisión o curso leve; 2) actividad inicial leve con posterior aumento en la severidad de los síntomas; 3 ) actividad crónica continua y 4) actividad crónica intermitente. Según resultados de una cohorte poblacional noruega (IBSEN) que incluyó 519 pacientes con CU seguidos por 10 años, $55 \%$ tuvo un curso tipo 1 , seguido por $37 \%$ con actividad crónica intermitente, $6 \%$ crónica continua y sólo 4 pacientes con actividad tipo $2^{5}$.

Los sistemas de clasificación en CU (Truelove-Witts [1955] ${ }^{6}$, Índice de Mayo [IMa] [1987] ${ }^{7}$ y la clasificación de Montreal $\left.[2005]^{8}\right)$ (Tabla 1), miden actividad en un momento determinado, siendo útiles en predecir la evolución en el corto plazo, pero no a largo plazo?

Esta revisión tiene por objetivo detallar factores predictivos, a corto y largo plazo, que permitan un diagnóstico riguroso y precoz, que favorezca la toma de decisiones terapéuticas, y así un tratamiento oportuno y eficaz. 
Tabla 1. Sistemas de clasificación en colitis ulcerosa

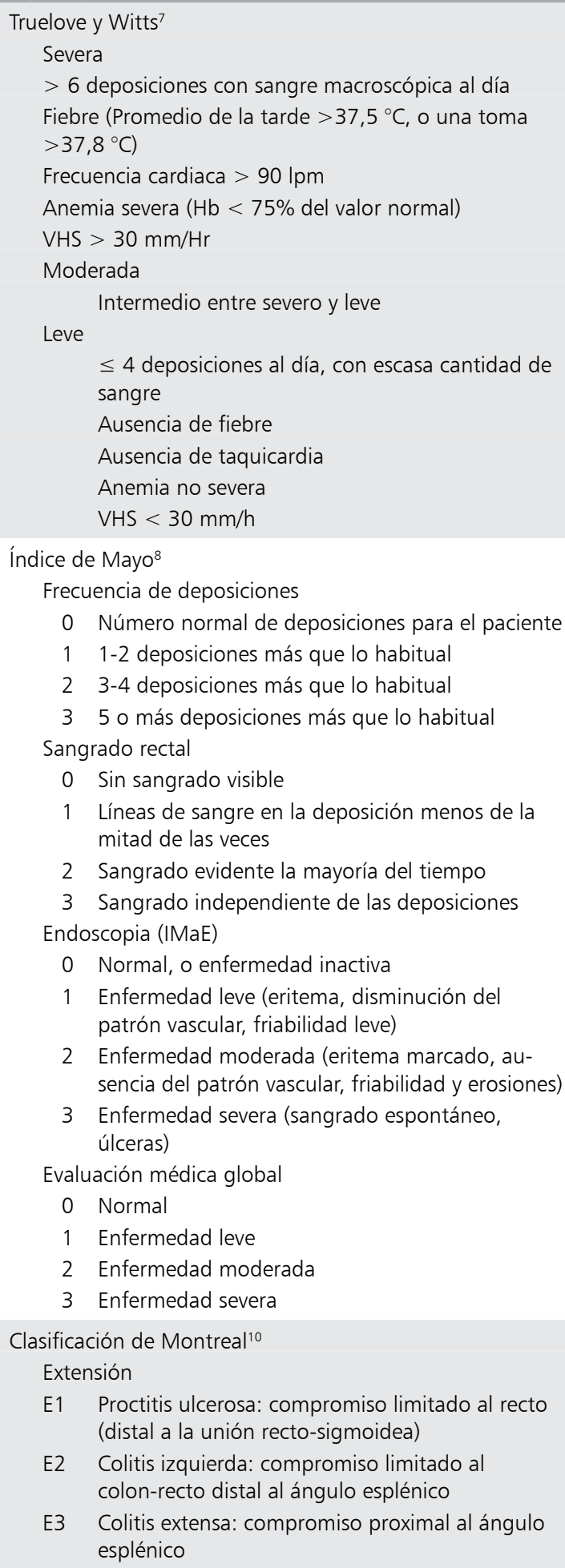

2 Sangrado evidente la mayoría del tiempo

3 Sangrado independiente de las deposiciones Endoscopia (IMaE)

0 Normal, o enfermedad inactiva

1 Enfermedad leve (eritema, disminución del patrón vascular, friabilidad leve)

2 Enfermedad moderada (eritema marcado, ausencia del patrón vascular, friabilidad y erosiones)

3 Enfermedad severa (sangrado espontáneo, úlceras)

Evaluación médica global

0 Normal

1 Enfermedad leve

2 Enfermedad moderada

3 Enfermedad severa

Clasificación de Montreal ${ }^{10}$

Extensión

E1 Proctitis ulcerosa: compromiso limitado al recto (distal a la unión recto-sigmoidea)

E2 Colitis izquierda: compromiso limitado al colon-recto distal al ángulo esplénico

E3 Colitis extensa: compromiso proximal al ángulo esplénico

\section{Severidad}

So Remisión clínica: Asintomático

S1 CU leve: cuatro o menos deposiciones al día (con o sin sangre), ausencia de cualquier enfermedad sistémica, y parámetros inflamatorios normales (VHS)

S2 CU Moderada: más de cuatro deposiciones por día, con mínimos signos de toxicidad sistémica

S3 CU Severa: al menos seis deposiciones con sangre al día, frecuencia cardiaca $\geq 90$ latidos por minuto, temperatura mínima de $37,5^{\circ} \mathrm{C}$, hemoglobina inferior a $10,5 \mathrm{~g} / \mathrm{dl}$, y VHS $\geq 30 \mathrm{~mm} / \mathrm{h}$

Índice de severidad endoscópico en Colitis Ulcerosa (UCEIS)

Patrón vascular

0 Normal Patrón vascular normal con arbolización de los capilares claramente definida o con perdida borrosa o parchada de los márgenes de los capilares

1 Obliteración parchada

2 Obliteración

Sangrado

$0 \quad$ Sin sangrado

1 Sangrado mucoso

2 Sangrado luminal leve

3 Sangrado luminal moderadosevero

Erosiones y úlceras

0 Normal

1 Erosiones

2 Úlceras superficiales

3 Úlceras prosundas
Obliteración parchada del patrón vascular

Obliteración completa del patrón vascular

Sin sangrado visible

Algunos puntos o restos de sangre coagulada en la superficie mucosa que no se logran lavar adecuadamente

Escasa cantidad de sangre líquida en el lúmen

Sangre evidente en el lumen por delante del endoscopio o sangrado en napa después de lavar, o sangrado persistente desde una mucosa hemorrágica

mucosa normal, sin erosiones o úlceras visibles

Defectos diminutos ( $5 \mathrm{~mm}$ ) en la mucosa, de color blanco o amarillo con un borde plano

Defectos en la mucosa más grandes (> $5 \mathrm{~mm}$ ): úlceras pequeñas cubiertas por fibrina pero que permanecen superficiales

Defectos mucosos más profundos, excavados con un borde ligeramente elevado 


\section{¿Cuáles son los factores pronósticos más importantes que se deben considerar en un paciente con CU?}

\section{Factores demográficos}

\section{Edad}

Pacientes jóvenes al diagnóstico presentan con mayor frecuencia períodos intercrisis de menor duración, mayor tasa de recaídas, menor respuesta al tratamiento, mayor severidad de la enfermedad, mayor probabilidad de extensión de la enfermedad y un mayor riesgo de colectomía ${ }^{5,10-15}$. Según datos de una cohorte de pacientes pediátricos con diagnóstico reciente de CU, la tasa de colectomía a 5 años fue de $20 \%{ }^{16}$. En el otro extremo, en un seguimiento de 472 pacientes $>60$ años al momento del diagnóstico, la probabilidad de colectomía a 10 años fue $8 \%{ }^{17}$. En el estudio IBSEN, los pacientes $>50$ años al diagnóstico, presentaron un riesgo 70\% menor de colectomía que aquellos diagnosticados antes de los 30 años $^{5}$. En otro registro prospectivo, con 3.752 pacientes seguidos entre 1984-2008, los pacientes $<25$ años al momento del diagnóstico presentaron mayores tasas de colectomía tardía (> 90 días desde la fecha de diagnóstico) que los $>65$ años (HR: 3,30, IC 2,08-5,24, $\mathrm{p}<0,0001)$. Al contrario, pacientes $>65$ años al momento del diagnóstico tuvieron una mayor probabilidad de colectomía temprana (<90 días desde la fecha de diagnóstico) (HR: 2.00, IC $1,33-3,55, \mathrm{p}=0,0079)^{11}$.

En suma, aunque una edad mayor al diagnóstico sería un factor protector de una enfermedad más severa, en aquellos que la presentan la posibilidad de colectomía temprana es mayor, probablemente por menor utilización de inmunosupresores o terapia biológica en esta población ${ }^{18,19}$.

\section{Tabaquismo}

Los mecanismos por los que este hábito disminuye la susceptibilidad, y determina un mejor pronóstico no están aclarados. Se sugiere que éste sería un factor "ambiental protector"20, proponiendo que la retirada del efecto inmunosupresor del tabaquismo, gatillaría la aparición de la enfermedad en un individuo genéticamente susceptible $^{21}$.

Estudios muestran que, a cualquier edad, ex-fumadores tienen mayor probabilidad de ser diagnosticados con $\mathrm{CU}$ que fumadores activos (HR 1,8, IC 1,41-2,44, p < 0,001). En el segui- miento a 5 años, se observó que la regresión de la extensión es más frecuente en fumadores activos que en ex-fumadores o no fumadores (EFNF) (30 vs 8 vs $5 \%, p<0,001)$. Aquellos pacientes con mayor consumo $(9,18$ paquetes/año, mediana) tuvieron menor compromiso, en extensión, que fumadores con menor consumo ( 0,320 paquete/ año (mediana), $\mathrm{p}=0,006)^{20}$. El grupo colaborativo europeo de estudio de enfermedades inflamatorias intestinales (EC-IBD) realizó un seguimiento prospectivo durante 10 años, a 781 pacientes con CU. El riesgo de recaída fue significativamente menor para fumadores activos que para EFNF (RR 0,8 , IC $0,6-0,9)^{10}$. Por otra parte, pacientes fumadores tendrían menor riesgo de colectomía (OR 0,55, IC 0,33-0,91) $)^{22}$.

\section{Género}

En la cohorte del EC-IBD, se observó que las mujeres presentan un mayor riesgo de recaídas (HR 1,2, IC: $1,1-1,3 ; \mathrm{p}<0,001)^{10}$. Aunque esta serie, mostró que el género no influenciaría las tasas de colectomía, un reciente metanálisis que incluyó 20 artículos confirma que las mujeres tendrían un menor riesgo de cirugía (OR 0,78; IC 0,68-0,90) 22 . Estudios prospectivos deberán confirmar estos resultados permitiendo definir los mecanismos que expliquen estas posibles diferencias.

Por otra parte, estudios han demostrado que las mujeres presentan una peor calidad de vida relacionada con la EII, especialmente en el aspecto emocional, lo que sugiere que el área psicológica cumple un rol importante en la terapia de la $\mathrm{EII}^{23,24}$.

\section{Cuadro clínico (severidad de la crisis)}

En 1996 Travisy cols., evaluaron 36 variables, en 51 episodios de CU grave, desarrollando un índice de predicción de colectomía (Oxford); 85\% requirió colectomía si al $3^{\text {er }}$ día de tratamiento (hidrocortisona ev y tópico) presentaban $>8$ deposiciones/día, o 3-8 deposiciones/día + PCR $>45 \mathrm{mg} / \mathrm{L}^{25}$. En 2004, Ho y cols. Publicaron los resultados de 167 pacientes con crisis grave de $\mathrm{CU}$. Este estudio propone la dilatación $>5,5 \mathrm{~cm}$ en colon transverso (radiografía de abdomen), albúmina sérica al ingreso $<3 \mathrm{~g} / \mathrm{dl}$ y la frecuencia defecatoria promedio (número de evacuaciones los días 1-3) como los principales factores predictores de falta de respuesta a tratamiento médico estándar (esteroides sistémicos, 5-ami- 
nosalicilatos) en $72 \mathrm{~h}$. Los autores proponen un índice que discrimina entre una probabilidad baja $(11 \%)$, intermedia $(45 \%)$ y alta $(85 \%)$ de fracaso a terapia; sugiriendo para los casos de riesgo elevado un tratamiento de rescate (ciclosporina (CsA) endovenosa o cirugía ${ }^{26}$. Ananthakrishnan y cols., sugieren un índice simplificado del riesgo de colectomía para pacientes hospitalizados por una crisis de $\mathrm{CU}$, dividiéndolos en tres grupos (alto, mediano y bajo riesgo), de acuerdo a una puntuación basada en datos clínicos; destacando el compromiso nutricional y la extensión del área comprometida ${ }^{27}$. Solberg y cols. desde la cohorte IBSEN, reconocen variables independientemente asociadas con riesgo de colectomía, según parámetros identificados al diagnóstico (extensión (E1-E2 vs E3), edad (< vs > 40 años), necesidad de esteroides sistémicos, proteína $C$ reactiva (PCR) o velocidad de sedimentación $(\mathrm{VHS})(<\mathrm{vs}>30)$, que combinándolos en una matriz de predicción, discrimina entre una probabilidad de colectomía de $2,6 \%$ a $40,1 \%$ a 10 años de evolución ${ }^{28}$ (Tabla 2). Una serie prospectiva, con 296 pacientes seguidos luego de inducida la remisión, reveló que al diagnóstico una crisis moderada-severa son factores independientes de colectomía tardía y de recaída. Niveles bajos de hemoglobina y albúmina, cuatro semanas posteriores a la inducción de la remisión, se asociaron con recaída y colectomía tardía. La presencia de una respuesta parcial al tratamiento de inducción predice recaída y colectomía tardía ${ }^{29}$. Otro registro retrospectivo, con pacientes hospitalizados por crisis de CU, concluyó que la tasa de colectomía electiva ha disminuido, en paralelo con la mayor utilización de inmunomoduladores (azatioprina/6-mercaptopurina ) y terapia biológica (infliximab (IFX)). Sin embargo, la tasa de colectomía de emergencia persiste estable ${ }^{30}$.

\section{Marcadores biológicos}

\section{Proteina $C$ reactiva ( $P C R$ )}

La PCR en la fase aguda de la respuesta inflamatoria, aumenta su producción con el aumento de interleucina (IL)-6, IL1 $\beta$ y TNF $\alpha^{31}$. Henriksen y cols., midieron, en 454 pacientes con $\mathrm{CU}$, niveles de PCR al diagnóstico, al año y 5 años de seguimiento; siendo directamente proporcionales con la extensión al momento del diagnóstico. El riesgo de colectomía en pacientes con CU extensa y PCR $>23 \mathrm{mg} / \mathrm{l}$, al diagnóstico, fue casi cinco veces ma- yor (OR 4,8, IC 1,5-15,1, p = 0,02); y aquellos que mantienen niveles $>10 \mathrm{mg} / \mathrm{l}$ al año de seguimiento presentan tres veces mayor riesgo de cirugía en los siguientes 4 años (OR 3,0, IC 1,1-7,8, $\mathrm{p}=0,02)^{32}$. Sandborn y cols, publican los datos referentes al riesgo de colectomía en los estudios ACT1 y 2; una PCR $>20 \mathrm{mg} / \mathrm{dl}$ al diagnóstico se asocia con mayor riesgo, independiente del tratamiento recibido $(\mathrm{HR} 1,73 \text {, IC 1,04-2,88, } \mathrm{p}=0,04)^{33}$.

\section{Calprotectina fecal (CF)}

D'Haens y cols. demostraron correlación entre los niveles de CF y la severidad de la actividad endoscópica (subíndice endoscópico de Mayo (IMaE), extensión de la enfermedad y actividad clínica (IMa)). Un corte de $250 \mu \mathrm{g} / \mathrm{g}$ mostró sensibilidad/especificidad de 71/85,7\% y $100 / 77,8 \%$ para "cualquier grado de inflamación" (IMaE 1-2 vs 0 ) y para inflamación "moderada-severa" (IMaE 2-3), respectivamente ${ }^{34}$. En otro estudio,con 90 pacientes con crisis grave, los valores de $\mathrm{CF}$ fueron significativamente mayores en pacientes con fracaso a tratamiento médico intensivo (esteroides \pm IFX) vs aquellos que no necesitaron cirugía ( $1.200 \mathrm{vs} 887 \mu \mathrm{g} / \mathrm{g}$ ). El $87 \%$ de los pacientes con CF $>1.922 \mu \mathrm{g} / \mathrm{gr}$ necesitaron colectomía en los seis meses posteriores. Al combinar CF sobre este valor, albumina $<3,4 \mathrm{~g} / \mathrm{dl}$ y PCR $>53 \mathrm{mg} / \mathrm{dl}$ se obtiene una sensibilidad de $66-70 \%$ para predecir colectomía durante la hospitalización ${ }^{35}$.

\section{Marcadores serológicos}

Anticuerpos anti-citoplasma de neutrófilos (ANCA), clásicamente asociados con vasculitis ${ }^{36}$, están presentes en $20-85 \%$ de los pacientes con $\mathrm{CU}$, específicamente con un patrón perinuclear $o$ atípico (pANCA) ${ }^{37}$.

Un estudio nacional determinó una prevalencia de $44 \%$ y $6 \%$ para pANCA y anticuerpos anti-Saccharomyces cerevisiae (ASCA), respectivamente. La presencia de pANCA se correlacionó significativamente con CU de $<5$ años de evolución,mayor número de crisis y un mayor porcentaje de colectomías ${ }^{38}$.

En un estudio retrospectivo, pANCA mostró asociación con mayor tasa de recaída (43 vs $27 \%)^{39}$. Otros han relacionado pANCA (+) con falla a tratamiento médico y necesidad de cirugía en CU izquierda, sugiriendo la necesidad de tratamiento inmunomodulador (IM) temprano ${ }^{40}$. En una serie prospectiva, en que se indujo remisión 
Tabla 2. Índices de riesgo de colectomía

\begin{tabular}{|c|c|c|c|c|c|}
\hline \multicolumn{6}{|l|}{ Ananthakrishnan y cols. ${ }^{14}$} \\
\hline \multicolumn{2}{|l|}{ Características al ingreso } & \multicolumn{4}{|l|}{ Puntuación } \\
\hline \multicolumn{2}{|l|}{ Anemia } & \multicolumn{2}{|l|}{1} & & \\
\hline \multicolumn{2}{|l|}{ Requerimiento de transfusión } & \multicolumn{2}{|l|}{1} & & \\
\hline \multicolumn{2}{|l|}{ Desnutrición } & \multicolumn{2}{|l|}{2} & & \\
\hline \multicolumn{2}{|l|}{ Nutrición parenteral total } & \multicolumn{2}{|l|}{1} & & \\
\hline \multicolumn{2}{|l|}{ Extensión E3 } & \multicolumn{2}{|l|}{2} & & \\
\hline \multicolumn{2}{|l|}{ Traslado desde otro centro } & \multicolumn{2}{|l|}{1} & & \\
\hline \multicolumn{2}{|l|}{ Admisión en hospital docente } & \multicolumn{2}{|l|}{1} & & \\
\hline \multicolumn{2}{|l|}{ Total } & \multicolumn{2}{|l|}{$0-9$} & & \\
\hline \multicolumn{6}{|c|}{ Estratificación de riesgo de colectomía: bajo ( 0-1) 1,4\%, intermedio ( $2-3$ ) 4,5\%, y alto (> 4) 15,6\%. } \\
\hline \multirow{2}{*}{\multicolumn{2}{|c|}{ Solberg y cols. ${ }^{15}$}} & \multicolumn{3}{|c|}{ PCR (mg/L) o VHS (mm/Hr) } & \\
\hline & & $<30$ & $\geq 30$ & & \\
\hline \multirow{4}{*}{ Edad } & $<40$ & $8,40 \%$ & $40,10 \%$ & Sí & \multirow{2}{*}{ Uso de esteroides } \\
\hline & $\geq 40$ & $2,60 \%$ & $16,50 \%$ & No & \\
\hline & & E1-2 & E3 & & \\
\hline & \multicolumn{4}{|c|}{ Extensión de la enfermedad } & \\
\hline
\end{tabular}

con IFX, aquellos pacientes pANCA(+)/ASCA(-) tuvieron significativamente menor respuesta clínica a las 10 semanas $^{41}$. En otro estudio, pANCA(-) se identificó como predictor independiente de sobrevida libre de recaída, posterior a inducción con IFX ${ }^{42}$.

\section{Marcadores genéticos}

Se han identificado diferentes loci asociados con riesgo de colectomía y progresión de la extensión (regiones HLA, IL1B, MDR1 y loci HSP70), como también un polimorfismo de nucleótido único asociado con respuesta favorable a IFX (IL23R) y otra variante protectora de CU grave (ATG16L1) ${ }^{43}$.

Iliev y cols. estudiaron la asociación entre el gen humano de Dectin-1 (receptor de inmunidad innata para hongos), CLEC7A y EII. Compararon alelos de CLEC7A en pacientes con CU refractaria a tratamiento médico y pacientes que no requirieron colectomía; el haplotipo $(\mathrm{AG})$ se relacionó con $\mathrm{CU}$ refractaria a tratamiento médico $(\mathrm{p}=0,0005)$ y menor tiempo para colectomía ${ }^{44}$.

Waterman y cols. realizaron un estudio de marcadores genéticos en base a un Inmunochip (196.524 polimorfismos), demostrando que ningún polimorfismo se asoció con severidad de la $\mathrm{CU}$, extensión proximal de la enfermedad o colectomía ${ }^{43}$.

Aunque esta es un área de creciente desarrollo y amplio potencial; es necesario estudios con una mayor cantidad de pacientes y poblaciones étnicas diferentes para confirmar relaciones significativas que permitan su uso clínico.

\section{Factores pronósticos endoscópicos}

Se han propuesto, al menos, nueve índices para determinar la severidad del compromiso mucoso en $\mathrm{CU}^{45}$. Destacan el $\mathrm{IMaE}^{7}$ y el Índice de severidad endoscópico (UCEIS), siendo este último el único índice validado ${ }^{46}$ (Tabla 1).

Carbonnel y cols., mostraron que en $43 / 46$ (93\%) pacientes con CU grave y lesiones graves (úlceras extensas y profundas) al ingreso requirieron cirugía, vs 10/39 (26\%) sin lesiones graves (OR $41)^{45,47}$. Dapermo y cols. demostraron una asociación significativa de menor respuesta a esteroides en presencia de úlceras profundas y pérdida extensa de la mucosa (OR 20,64, IC: 4,5-94,15, $\mathrm{p}<0,0001)^{48}$. Otro estudio señala que un UCEIS $\geq 7 / 8$ al ingreso requiere tratamiento de rescate (CsA/IFX) en $79 \%{ }^{49}$. La extensión del compromiso también se asocia con mayor riesgo de colectomía; 19\% en 10 años para CU extensa versus $5 \%$ para proctitis ${ }^{5}$. 


\section{Curación de la mucosa}

La Organización Internacional de Enfermedad Inflamatoria Intestinal definió curación de la mucosa (CM) en CU como la ausencia de friabilidad, sangre, erosiones y úlceras en todos los segmentos visualizados de la mucosa del colon ${ }^{50}$. Sin embargo, diferentes estudios han permitido la presencia de eritema y friabilidad en esta definición ${ }^{51}$. Froslie y cols., siguieron por 5 años a 354 pacientes con $\mathrm{CU}$; aquellos con IMaE 0 , al año de tratamiento, presentan un menor riesgo de colectomía (RR $0,22$, IC $0,06-0,79, p=0,02)^{52}$. Colombel y cols., reportan que los pacientes que logran CM temprana (IMaE 0-1 en la semana 8 de tratamiento con IFX) presentan, en forma significativa, una mayor probabilidad de mantenerse libre de colectomía (95\% vs $80 \%$ IMaE 3; $p=0,0004$ ), y una mayor probabilidad de mantener remisión clínica (73$47 \%$ vs $10 \% \mathrm{IMaE} 3 ; \mathrm{p}<0,0001)$ y CM $(77-54 \%$ vs $6,7 \% \operatorname{IMaE} 3 ; \mathrm{p}<0,0001)$ a la semana $54^{53}$.

Otro estudio señala que el riesgo de CCR, en CU extensa que alcanzan CM, sería el mismo que en población general ${ }^{54}$. En un seguimiento de un año, a 138 pacientes en remisión libre de esteroides (44,2\% IMaE 0 vs $55,8 \% \mathrm{IMaE} 1)$, aquellos con IMaE 1 presentaron un riesgo tres veces mayor de recaída $(\mathrm{p}=0,026)^{55}$. Desde 2011 la CM emerge como una meta importante en el tratamiento ${ }^{56}$, proponiéndose escalar en la terapia en búsqueda de este objetivo ${ }^{57}$.

\section{Predictores histológicos}

Dado que la curación endoscópica de la mucosa no es un indicador preciso de curación histológica, y 16-100\% de los pacientes en remisión endoscópica presentarían inflamación histológi$\mathrm{ca}^{58}$, la evaluación histológica se está convirtiendo en un objetivo potencial, no sólo para evaluar la actividad de la CU, sino para predecir su evolución y tratamiento ${ }^{59}$. La remisión histológica sería mejor predictor de menor uso de corticoesteroides y necesidad de hospitalización por crisis grave al compararla con remisión endoscópica (HR 0,42, $0,2-0,9, \mathrm{p}=0,02$; HR $0,21,0,1-0,7, \mathrm{p}=0,02$; respectivamente) ${ }^{60}$.

Se han propuesto diversos índices para evaluar el grado de actividad histológica en CU, todos con un adecuado grado de correlación ${ }^{61}$. Los más utilizados son el índice de Riley y el de Geboes, este último incorpora seis criterios que evalúan inflamación aguda y crónica ${ }^{62}$. Bessissow y cols, re- trospectivamente estudian 75 pacientes con IMaE0 en tratamiento de mantención con mesalazina, IM y/o biológico. Un $40 \%$ presentó un índice Geboes $\geq 3,1$ (enfermedad activa) y $21 \%$ presentó plasmocitosis basal (asociado con recaída temprana ${ }^{12}$ ). En el análisis multivariado, plasmocitosis basal representó 5 veces mayor riesgo de recaída en un año (IC 1,32-19,99, p = 0,019) ${ }^{63}$.Estos resultados confirman el rol de la actividad histológica en la evolución de la CU.

Estudios han planteado una adecuada correlación entre histología y endocitoscopia ${ }^{59}$, sugiriendo esta técnica como una herramienta útil en la predicción de recaída ${ }^{64}$.

\section{Co-infecciones}

Aunque la mayoría de los estudios relacionados con infección intestinal en EII han sido enfocados a Clostridium difficile y Citomegalovirus, otros han planteado evaluar el rol de otras infecciones intestinales en su desarrollo y evolución ${ }^{65}$.

\section{Citomegalovirus}

Es fundamental descartar su presencia en pacientes con CU grave, especialmente en aquellos que se encuentran en tratamiento con esteroides/ inmunosupresores ${ }^{66-68}$. Un estudio cohorte retrospectivo de 10 años, determinó una prevalencia de $22,7 \%$ en CU y $16 \%$ en EC. Esta infección se asoció significativamente con las siguientes variables al ingreso: edad $>30$ años (OR 14,26, IC 2,89-118,57), duración de la enfermedad $<5$ años (OR 7,69, IC 1,80-45,41), tratamiento inmunosupresor (IM \pm IFX o ADA) (OR 6,73, IC $1,67-35,63$ ) y leucocitos $<11.000$ (OR 4.49, IC1,15-21,79) ${ }^{69}$. La presencia de Citomegalovirus durante una crisis grave se ha asociado significativamente con corticorefractariedad (OR 2.28, IC $1,10-4,72, p=0,026)^{70}$. Un recuento de $\geq 5$ células positivas para inmunohistoquímca por muestra de biopsia se asociaría, de manera significativa, con mayores tasas de colectomía ${ }^{71}$. Por otro lado, se han observado altas tasas de remisión con tratamiento antiviral $(71-81 \%)^{72}$.

\section{Clostridium difficile}

La infección por Clostridium difficile (ICD) presenta una mayor incidencia en pacientes con EII en crisis, particularmente en $\mathrm{CU}^{73}$, incidiendo su pronóstico a corto y mediano plazo ${ }^{74-78}$. Se des- 
criben factores de riesgo: 1) uso de antibióticos: su uso en los últimos 30 días representa un OR de 12 (IC 1,2-124,2) ${ }^{79}$, sin embargo, la ausencia de exposición a antibióticos no debe limitar su estudio; 2) Inmunosupresión: Schneeweiss y cols. determinan un riesgo tres veces mayor de ICD asociado al uso de esteroides, pero no a otros IM o IFX ${ }^{80}$.

La ICD se ha asociado en el corto plazo con mayores costos de tratamiento, estadía hospitalaria y riesgo de colectomía ${ }^{81}$. Al año de seguimiento, se asociaría a mayor número de hospitalizaciones y consultas en servicio de urgencia por crisis de $\mathrm{CU}^{82}$. Un estudio de caso-control mostró que posterior a una ICD 53\% de los pacientes requirió modificaciones en el tratamiento ${ }^{83}$. Un diagnóstico precoz y un tratamiento adecuado de esta infección es relevante para mejorar el resultado en pacientes con $\mathrm{CU}^{84}$.

\section{Colangitis esclerosante primaria (CEP)}

La CEP se identificó como factor predictor de progresión de la extensión en un estudio prospectivo con 420 pacientes (HR 12,83, IC 1,36-121,10) seguidos por 10 años $^{14}$. Broome y cols. demostraron un riesgo acumulado de CCR en pacientes con CU y CEP de 9\%, 31\% y hasta 50\% en 10, 20 y 25 años; en comparación con $2 \%, 5 \%$ y $10 \%$ en pacientes sólo con $\mathrm{CU}^{85}$. Un meta-análisis mostró que un $21 \%$ de los pacientes con CU y CEP desarrolló CCR vs 4\% en pacientes sin CEP (OR 4,79, IC $3,58-6,41)^{86}$. Por lo referido, se recomienda vigilancia endoscópica desde su diagnóstico y anualmente en forma indefinida ${ }^{87}$.

\section{Conclusión}

La CU es una enfermedad heterogénea en su desarrollo y evolución siendo importante identificar sobre todo al diagnóstico aquellos pacientes que presentarán un curso rápido y severo. Esta evaluación permitirá definir precozmente aquellos pacientes que deberán iniciar una terapia más agresiva (IM y/o terapia biológica) disminuyendo el riesgo de complicaciones y colectomía. Aunque múltiples variables han sido consideradas al momento de definir el pronóstico de estos pacientes (Tabla 3), la mayoría han sido definidas desde estudios retrospectivos. Las variables más importantes parecen ser el antecedente de hos-
Tabla 3. Factores pronósticos en CU de reciente diagnóstico

Demográficos
Edad
Género
Tabaquismo
Cuadro clínico
Marcadores biológicos
Proteína C reactiva
Calprotectina fecal
Marcadores serológicos
pANCA
ASCA
Marcadores genéticos
Marcadores endoscópicos
Curación de la mucosa
Predictores histológicos
Co-infecciones
Clostridium difficile
Citomegalovirus
Colangitis esclerosante primaria

pitalización precoz al diagnóstico, niveles bajos de hemoglobina, valores elevados de PCR y CF, presencia de úlceras profundas en la colonoscopia, sobreinfección agregada y ausencia de curación de la mucosa durante la evolución. Estudios prospectivos son necesarios para validar estos factores pronósticos o definir si otras variables como la microbiota tienen algún rol en la evolución de estos pacientes.

\section{Referencias}

1. Sepúlveda SE, Beltrán CJ, Peralta A, Rivas P, Rojas N, Figueroa C, et al. [Inflammatory bowel diseases: an immunological approach]. Rev Med Chile 2008; 136 (3): 367-75.

2. Ordas I, Eckmann L, Talamini M, Baumgart DC, Sandborn WJ. Ulcerative colitis. Lancet (London, England). 2012; 380 (9853): 1606-19.

3. Simian D, Fluxá D, Flores L, Lubascher J, Ibáñez P, Figueroa $\mathrm{C}$, et al. Inflammatory bowel disease: A descriptive study of 716 local Chilean patients. World journal of gastroenterology 2016; 22 (22): 5267-75.

4. Brostrom O. Prognosis in ulcerative colitis. The Medical clinics of North America 1990; 74 (1): 201-18. 
5. Solberg IC, Lygren I, Jahnsen J, Aadland E, Hoie O, Cvancarova $\mathrm{M}$, et al. Clinical course during the first 10 years of ulcerative colitis: results from a population-based inception cohort (IBSEN Study). Scandinavian journal of gastroenterology 2009; 44 (4): 431-40.

6. Truelove SC, Witts LJ. Cortisone in ulcerative colitis; final report on a therapeutic trial. British Medical Journal 1955; 2 (4947): 1041-8.

7. Schroeder KW, Tremaine WJ, Ilstrup DM. Coated Oral 5-Aminosalicylic Acid Therapy for Mildly to Moderately Active Ulcerative Colitis. New England Journal of Medicine 1987; 317 (26): 1625-9.

8. Satsangi J, Silverberg MS, Vermeire S, Colombel JF. The Montreal classification of inflammatory bowel disease: controversies, consensus, and implications. Gut 2006; 55 (6): 749-53.

9. Silverberg MS, Satsangi J, Ahmad T, Arnott ID, Bernstein CN, Brant SR, et al. Toward an integrated clinical, molecular and serological classification of inflammatory bowel disease: report of a Working Party of the 2005 Montreal World Congress of Gastroenterology. Canadian journal of gastroenterology = Journal canadien de gastroenterologie 2005; 19 Suppl A: 5a-36a.

10. Hoie O, Wolters F, Riis L, Aamodt G, Solberg C, Bernklev $\mathrm{T}$, et al. Ulcerative colitis: patient characteristics may predict 10-yr disease recurrence in a European-wide population-based cohort. The American journal of gastroenterology 2007; 102 (8): 1692-701.

11. Targownik LE, Singh H, Nugent Z, Bernstein CN. The epidemiology of colectomy in ulcerative colitis: results from a population-based cohort. The American journal of gastroenterology 2012; 107 (8): 1228-35.

12. Bitton A, Peppercorn MA, Antonioli DA, Niles JL, Shah $\mathrm{S}$, Bousvaros A, et al. Clinical, biological, and histologic parameters as predictors of relapse in ulcerative colitis. Gastroenterology 2001; 120 (1): 13-20.

13. Roth LS, Chande N, Ponich T, Roth ML, Gregor J. Predictors of disease severity in ulcerative colitis patients from Southwestern Ontario. World journal of gastroenterology 2010; 16 (2): 232-6.

14. Etchevers MJ, Aceituno M, García-Bosch O, Ordas I, Sans M, Ricart E, et al. Risk factors and characteristics of extent progression in ulcerative colitis. Inflammatory bowel diseases 2009; 15 (9): 1320-5.

15. Ha CY, Newberry RD, Stone CD, Ciorba MA. Patients with late-adult-onset ulcerative colitis have better outcomes than those with early onset disease. Clinical gastroenterology and hepatology : the official clinical practice journal of the American Gastroenterological Association 2010; 8 (8): 682-7.e1.

16. Gower-Rousseau C, Dauchet L, Vernier-Massouille G,
Tilloy E, Brazier F, Merle V, et al. The natural history of pediatric ulcerative colitis: a population-based cohort study. The American journal of gastroenterology 2009; 104 (8): 2080-8.

17. Charpentier C, Salleron J, Savoye G, Fumery M, Merle V, Laberenne JE, et al. Natural history of elderly-onset inflammatory bowel disease: a population-based cohort study. Gut 2014; 63 (3): 423-32.

18. Lin WC, Tung CC, Lin HH, Lin CC, Chang CW, Yen $\mathrm{HH}$, et al. Elderly Adults with Late-Onset Ulcerative Colitis Tend to Have Atypical, Milder Initial Clinical Presentations but Higher Surgical Rates and Mortality: A Taiwan Society of Inflammatory Bowel Disease Study. Journal of the American Geriatrics Society 2016.

19. Shi HY, Chan FK, Leung WK, Li MK, Leung CM, Sze SF, et al. Natural History of Elderly-onset Ulcerative Colitis: Results from a Territory-wide Inflammatory Bowel Disease Registry. Journal of Crohn's \& colitis 2016; 10 (2): 176-85.

20. Aldhous MC, Drummond HE, Anderson N, Baneshi MR, Smith LA, Arnott ID, et al. Smoking habit and load influence age at diagnosis and disease extent in ulcerative colitis. The American journal of gastroenterology 2007; 102 (3): 589-97.

21. Abraham N, Selby W, Lazarus R, Solomon M. Is smoking an indirect risk factor for the development of ulcerative colitis? An age- and sex-matched case-control study. Journal of gastroenterology and hepatology 2003; 18 (2): 139-46.

22. Dias CC, Rodrigues PP, da Costa-Pereira A, Magro F. Clinical predictors of colectomy in patients with ulcerative colitis: systematic review and meta-analysis of cohort studies. Journal of Crohn's \& colitis 2015; 9 (2): 156-63.

23. Huppertz-Hauss G, Lie Hoivik M, Jelsness-Jorgensen LP, Henriksen M, Hoie O, Jahnsen J, et al. Health-related Quality of Life in Patients with Inflammatory Bowel Disease 20 Years After Diagnosis: Results from the IBSEN Study. Inflammatory bowel diseases 2016; 22 (7): 1679-87.

24. Zheng K, Zhang S, Wang C, Zhao W, Shen H. Health-related quality of life in Chinese patients with mild and moderately active ulcerative colitis. PLoS One 2015; 10 (4): e0124211.

25. Travis SP, Farrant JM, Ricketts C, Nolan DJ, Mortensen NM, Kettlewell MG, et al. Predicting outcome in severe ulcerative colitis. Gut 1996; 38 (6): 905-10.

26. Ho GT, Mowat C, Goddard CJ, Fennell JM, Shah NB, Prescott RJ, et al. Predicting the outcome of severe ulcerative colitis: development of a novel risk score to aid early selection of patients for second-line medical thera- 
py or surgery. Alimentary pharmacology \& therapeutics. 2004; 19 (10): 1079-87.

27. Ananthakrishnan AN, McGinley EL, Binion DG, Saeian K. Simple score to identify colectomy risk in ulcerative colitis hospitalizations. Inflammatory bowel diseases. 2010; 16 (9): 1532-40.

28. Solberg IC, Hoivik ML, Cvancarova M, Moum B. Risk matrix model for prediction of colectomy in a population-based study of ulcerative colitis patients (the IBSEN study). Scandinavian journal of gastroenterology 2015; 50 (12): 1456-62.

29. Shiga $\mathrm{H}$, Takagi $\mathrm{S}$, Inoue $\mathrm{R}$, Kinouchi $\mathrm{Y}$, Ohkubo $\mathrm{T}$, Takahashi S, et al. What determines the later clinical course of patients who do not undergo colectomy at the first attack? A Japanese cohort study on ulcerative colitis. Digestion 2010; 81 (2): 104-12.

30. Kaplan GG, Seow CH, Ghosh S, Molodecky N, Rezaie A, Moran GW, et al. Decreasing colectomy rates for ulcerative colitis: a population-based time trend study. The American journal of gastroenterology 2012; 107 (12): 1879-87.

31. Darlington GJ, Wilson DR, Lachman LB. Monocyte-conditioned medium, interleukin-1, and tumor necrosis factor stimulate the acute phase response in human hepatoma cells in vitro. The Journal of cell biology 1986; 103 (3): 787-93.

32. Henriksen M, Jahnsen J, Lygren I, Stray N, Sauar J, Vatn $\mathrm{MH}$, et al. C-reactive protein: a predictive factor and marker of inflammation in inflammatory bowel disease. Results from a prospective population-based study. Gut 2008; 57 (11): 1518-23.

33. Sandborn WJ, Rutgeerts P, Feagan BG, Reinisch W, Olson A, Johanns J, et al. Colectomy rate comparison after treatment of ulcerative colitis with placebo or infliximab. Gastroenterology 2009; 137 (4): 1250-60; quiz 520.

34. D'Haens G, Ferrante M, Vermeire S, Baert F, Noman $\mathrm{M}$, Moortgat L, et al. Fecal calprotectin is a surrogate marker for endoscopic lesions in inflammatory bowel disease. Inflammatory bowel diseases 2012; 18 (12): 2218-24.

35. Ho GT, Lee HM, Brydon G, Ting T, Hare N, Drummond $\mathrm{H}$, et al. Fecal calprotectin predicts the clinical course of acute severe ulcerative colitis. The American journal of gastroenterology 2009; 104 (3): 673-8.

36. Mendoza JL, Abreu MT. Biological markers in inflammatory bowel disease: practical consideration for clinicians. Gastroenterologie clinique et biologique 2009; 33 Suppl 3: S158-73.

37. Reese GE, Constantinides VA, Simillis C, Darzi AW, Orchard TR, Fazio VW, et al. Diagnostic precision of
anti-Saccharomyces cerevisiae antibodies and perinuclear antineutrophil cytoplasmic antibodies in inflammatory bowel disease. The American journal of gastroenterology 2006; 101 (10): 2410-22.

38. Vergara AT, Cofré LP, Cifuentes AS, Pulgar AU, Puebla AC, Velasco PS. Prevalencia de marcadores serológicos ANCA y ASCA en una población con colitis ulcerosa. Rev Med Chile 2006; 134: 960-4.

39. Lombardi G, Annese V, Piepoli A, Bovio P, Latiano A, Napolitano G, et al. Antineutrophil cytoplasmic antibodies in inflammatory bowel disease: clinical role and review of the literature. Diseases of the colon and rectum 2000; 43 (7): 999-1007.

40. Abreu MT. Controversies in IBD. Serologic tests are helpful in managing inflammatory bowel disease. Inflammatory bowel diseases 2002; 8 (3): 224-6; discussion 3, 30-1.

41. Ferrante M, Vermeire S, Katsanos KH, Noman M, Van Assche G, Schnitzler F, et al. Predictors of early response to infliximab in patients with ulcerative colitis. Inflammatory bowel diseases 2007; 13 (2): 123-8.

42. Arias MT, Vande Casteele N, Vermeire S, de Buck van Overstraeten A, Billiet T, Baert F, et al. A panel to predict long-term outcome of infliximab therapy for patients with ulcerative colitis. Clinical gastroenterology and hepatology : the official clinical practice journal of the American Gastroenterological Association 2015; 13 (3): 531-8.

43. Waterman M, Knight J, Dinani A, Xu W, Stempak JM, Croitoru K, et al. Predictors of Outcome in Ulcerative Colitis. Inflammatory bowel diseases 2015; 21 (9): 2097 105.

44. Iliev ID, Funari VA, Taylor KD, Nguyen Q, Reyes CN, Strom SP, et al. Interactions between commensal fungi and the C-type lectin receptor Dectin-1 influence colitis. Science (New York, NY) 2012; 336 (6086): 1314-7.

45. Allez M, Lemann M. Role of endoscopy in predicting the disease course in inflammatory bowel disease. World journal of gastroenterology 2010; 16 (21): 2626-32.

46. Travis SP, Schnell D, Krzeski P, Abreu MT, Altman DG, Colombel JF, et al. Developing an instrument to assess the endoscopic severity of ulcerative colitis: the Ulcerative Colitis Endoscopic Index of Severity (UCEIS). Gut 2012; 61 (4): 535-42.

47. Carbonnel F, Lavergne A, Lemann M, Bitoun A, Valleur P, Hautefeuille P, et al. Colonoscopy of acute colitis. A safe and reliable tool for assessment of severity. Digestive diseases and sciences 1994; 39 (7): 1550-7.

48. Daperno M, Sostegni R, Scaglione N, Ercole E, Rigazio C, Rocca R, et al. Outcome of a conservative approach in severe ulcerative colitis. Digestive and liver disease: 
official journal of the Italian Society of Gastroenterology and the Italian Association for the Study of the Liver 2004; 36 (1): 21-8.

49. Corte C, Fernandopulle N, Catuneanu AM, Burger D, Cesarini M, White L, et al. Association between the ulcerative colitis endoscopic index of severity (UCEIS) and outcomes in acute severe ulcerative colitis. Journal of Crohn's \& colitis 2015; 9 (5): 376-81.

50. D'Haens G, Sandborn WJ, Feagan BG, Geboes K, Hanauer SB, Irvine EJ, et al. A review of activity indices and efficacy end points for clinical trials of medical therapy in adults with ulcerative colitis. Gastroenterology 2007; 132 (2): 763-86.

51. Dave M, Loftus EV, Jr. Mucosal healing in inflammatory bowel disease-a true paradigm of success? Gastroenterology \& hepatology 2012; 8 (1): 29-38.

52. Froslie KF, Jahnsen J, Moum BA, Vatn MH. Mucosal healing in inflammatory bowel disease: results from a Norwegian population-based cohort. Gastroenterology 2007; 133 (2): 412-22.

53. Colombel JF, Rutgeerts P, Reinisch W, Esser D, Wang Y, Lang Y, et al. Early mucosal healing with infliximab is associated with improved long-term clinical outcomes in ulcerative colitis. Gastroenterology 2011; 141 (4): 1194-201.

54. Rutter MD, Saunders BP, Wilkinson KH, Rumbles S, Schofield G, Kamm MA, et al. Cancer surveillance in longstanding ulcerative colitis: endoscopic appearances help predict cancer risk. Gut 2004; 53 (12): 1813-6.

55. Boal Carvalho P, Dias de Castro F, Rosa B, Moreira MJ, Cotter J. Mucosal Healing in Ulcerative Colitis - When Zero is Better. Journal of Crohn's \& colitis. 2015.

56. Peyrin-Biroulet L, Ferrante M, Magro F, Campbell S, Franchimont D, Fidder $\mathrm{H}$, et al. Results from the 2nd Scientific Workshop of the ECCO (I): Impact of mucosal healing on the course of inflammatory bowel disease. Journal of Crohn's and Colitis 2011; 5 (5): 477-83.

57. Walsh A, Palmer R, Travis S. Mucosal healing as a target of therapy for colonic inflammatory bowel disease and methods to score disease activity. Gastrointestinal endoscopy clinics of North America 2014; 24 (3): 367-78.

58. Bryant RV, Winer S, Spl T, Riddell RH. Systematic review: Histological remission in inflammatory bowel disease. Is 'complete' remission the new treatment paradigm? An IOIBD initiative. Journal of Crohn's and Colitis 2014; 8 (12): 1582-97.

59. Marchal Bressenot A, Riddell RH, Boulagnon-Rombi C, Reinisch W, Danese S, Schreiber S, et al. Review article: the histological assessment of disease activity in ulcerative colitis. Alimentary pharmacology \& therapeutics 2015; 42 (8): 957-67.
60. Bryant RV, Burger DC, Delo J, Walsh AJ, Thomas S, von Herbay A, et al. Beyond endoscopic mucosal healing in UC: histological remission better predicts corticosteroid use and hospitalisation over 6 years of follow-up. Gut 2015.

61. Bressenot A, Salleron J, Bastien C, Danese S, Boulagnon-Rombi C, Peyrin-Biroulet L. Comparing histological activity indexes in UC. Gut 2015; 64 (9): 1412-8.

62. Geboes K, Riddell R, Ost A, Jensfelt B, Persson T, Lofberg R. A reproducible grading scale for histological assessment of inflammation in ulcerative colitis. Gut 2000; 47 (3): 404-9.

63. Bessissow T, Lemmens B, Ferrante M, Bisschops R, Van Steen K, Geboes K, et al. Prognostic value of serologic and histologic markers on clinical relapse in ulcerative colitis patients with mucosal healing. The American journal of gastroenterology 2012; 107 (11): 1684-92.

64. Nishiyama S, Oka S, Tanaka S, Sagami S, Nagai K, Ueno $\mathrm{Y}$, et al. Clinical usefulness of endocytoscopy in the remission stage of ulcerative colitis: a pilot study. Journal of gastroenterology 2015; 50 (11): 1087-93.

65. Lobaton T, Domenech E. Bacterial Intestinal Superinfections in Inflammatory Bowel Diseases Beyond Clostridum difficile. Inflammatory bowel disease 2016; 22 (7): 1755-62.

66. Cottone M, Pietrosi G, Martorana G, Casa A, Pecoraro G, Oliva L, et al. Prevalence of cytomegalovirus infection in severe refractory ulcerative and Crohn's colitis. The American journal of gastroenterology 2001; 96 (3): 773-5.

67. Domenech E, Vega R, Ojanguren I, Hernández A, García-Planella E, Bernal I, et al. Cytomegalovirus infection in ulcerative colitis: a prospective, comparative study on prevalence and diagnostic strategy. Inflamm Bowel Dis 2008; 14 (10): 1373-9.

68. Figueroa C. Citomegalovirus en enfermedad inflamatoria intestinal. Gastr Latinoam 2013; 24 (Suplemento No1): S41-3.

69. Gauss A, Rosenstiel S, Schnitzler P, Hinz U, Rehlen T, Kadmon M, et al. Intestinal cytomegalovirus infection in patients hospitalized for exacerbation of inflammatory bowel disease: a 10-year tertiary referral center experience. European journal of gastroenterology \& hepatology 2015; 27 (6): 712-20.

70. Lee HS, Park SH, Kim SH, Kim J, Choi J, Lee HJ, et al. Risk Factors and Clinical Outcomes Associated with Cytomegalovirus Colitis in Patients with Acute Severe Ulcerative Colitis. Inflammatory bowel diseases 2016; 22 (4): 912-8.

71. Zagorowicz E, Bugajski M, Wieszczy P, Pietrzak A, Magdziak A, Mroz A. Cytomegalovirus Infection in Ulcerative Colitis is Related to Severe Inflammation and a High 
Count of Cytomegalovirus-positive Cells in Biopsy Is a Risk Factor for Colectomy. Journal of Crohn's \& colitis 2016; 10 (10): 1205-11.

72. Sager K, Alam S, Bond A, Chinnappan L, Probert CS. Review article: cytomegalovirus and inflammatory bowel disease. Alimentary pharmacology \& therapeutics 2015; 41 (8): 725-33.

73. Rodemann JF, Dubberke ER, Reske KA, Seo da H, Stone CD. Incidence of Clostridium difficile infection in inflammatory bowel disease. Clinical gastroenterology and hepatology: the official clinical practice journal of the American Gastroenterological Association 2007; 5 (3): 339-44.

74. Bernstein CN, Fried M, Krabshuis JH, Cohen H, Eliakim R, Fedail S, et al. World Gastroenterology Organization Practice Guidelines for the diagnosis and management of IBD in 2010. Inflammatory bowel diseases 2010; 16 (1): 112-24.

75. Bitton A, Buie D, Enns R, Feagan BG, Jones JL, Marshall JK, et al. Treatment of hospitalized adult patients with severe ulcerative colitis: Toronto consensus statements. The American journal of gastroenterology 2012; 107 (2): 179-94; author reply 95.

76. Gomollon F, García-López S, Sicilia B, Gisbert JP, Hinojosa J. [Therapeutic guidelines on ulcerative colitis: a GRADE methodology based effort of GETECCU]. Gastroenterologia y hepatologia 2013; 36 (8): e1-47.

77. Kornbluth A, Sachar DB. Ulcerative colitis practice guidelines in adults: American College Of Gastroenterology, Practice Parameters Committee. The American journal of gastroenterology 2010; 105 (3): 501-23; quiz 24.

78. Van Assche G, Vermeire S, Rutgeerts P. Management of acute severe ulcerative colitis. Gut 2011; 60 (1): 130-3.

79. Kariv R, Navaneethan U, Venkatesh PG, López R, Shen B. Impact of Clostridium difficile infection in patients with ulcerative colitis. Journal of Crohn's \& colitis 2011; 5 (1): 34-40.

80. Schneeweiss S, Korzenik J, Solomon DH, Canning C, Lee J, Bressler B. Infliximab and other immunomodulating drugs in patients with inflammatory bowel disease and the risk of serious bacterial infections. Alimentary pharmacology \& therapeutics 2009; 30 (3): 253-64.

81. Navaneethan U, Venkatesh PG, Shen B. Clostridium difficile infection and inflammatory bowel disease: understanding the evolving relationship. World journal of gastroenterology 2010; 16 (39): 4892-904.

82. Jodorkovsky D, Young Y, Abreu MT. Clinical outcomes of patients with ulcerative colitis and co-existing Clostridium difficile infection. Digestive diseases and sciences 2010; 55 (2): 415-20.

83. Chiplunker A, Ananthakrishnan AN, Beaulieu DB, Naik AS, Zadvornova Y, Skaros S, et al. Long-term impact of Clostridium difficile on inflammatory bowel disease. Gastroenterology 2009; 136 (Suppl 1): S1145.

84. Trifan A, Stanciu C, Stoica O, Girleanu I, Cojocariu C. Impact of Clostridium difficile infection on inflammatory bowel disease outcome: a review. World journal of gastroenterology 2014; 20 (33): 11736-42.

85. Broome U, Lofberg R, Veress B, Eriksson LS. Primary sclerosing cholangitis and ulcerative colitis: evidence for increased neoplastic potential. Hepatology (Baltimore, Md) 1995; 22 (5): 1404-8.

86. Soetikno RM, Lin OS, Heidenreich PA, Young HS, Blackstone MO. Increased risk of colorectal neoplasia in patients with primary sclerosing cholangitis and ulcerative colitis: a meta-analysis. Gastrointestinal endoscopy 2002; 56 (1): 48-54.

87. Farraye FA, Odze RD, Eaden J, Itzkowitz SH. AGA Technical Review on the Diagnosis and Management of Colorectal Neoplasia in Inflammatory Bowel Disease. Gastroenterology 138 (2): 746-74. 